\title{
EXPLICIT SOLUTIONS OF ONE-DIMENSIONAL, FIRST-ORDER, STATIONARY MEAN-FIELD GAMES WITH CONGESTION
}

\author{
Diogo A. Gomes, Levon Nurbekyan and Mariana Prazeres
}

\begin{abstract}
Here, we consider one-dimensional firstorder stationary mean-field games with congestion. These games arise when crowds face difficulty moving in highdensity regions. We look at both monotone decreasing and increasing interactions and construct explicit solutions using the current formulation. We observe new phenomena such as discontinuities, unhappiness traps and the nonexistence of solutions.
\end{abstract}

\section{INTRODUCTION}

Mean-field games (MFG) model interactions between an infinite number of identical rational agents. These games were independently introduced in [19], [20], [21] and in [17], [18]. MFGs are described by a system of differential equations: a Hamilton-Jacobi (HJ) equation for a value function, $u$, determining the agent's preferences and a Fokker-Plank (FP) equation for the population density, $m$.

We consider the one-dimensional, first-order, stationary MFG with congestion

$$
\left\{\begin{array}{l}
\frac{\left(u_{x}+p\right)^{2}}{2 m^{\alpha}}+V(x)=g(m(x))+\bar{H} \\
-\left(m^{1-\alpha}\left(u_{x}+p\right)\right)_{x}=0 .
\end{array}\right.
$$

Here, $\alpha \in \mathbb{R}$ encodes the congestion strength, $p \in \mathbb{R}$ a preferred direction of motion, $V: \mathbb{T} \rightarrow \mathbb{R}$ the spatial preference, where $\mathbb{T}$ is the one-dimensional torus. Furthermore, $g: \mathbb{R}^{+} \rightarrow \mathbb{R}$ gives additional meanfield interactions. Both $V$ and $g$ are $C^{\infty}$. A solution of (I.1) is a triplet $(u, m, \bar{H})$ where $u, m: \mathbb{T} \rightarrow \mathbb{R}$ and $\bar{H} \in \mathbb{R}$. We assume that $u$ is a Lipschitz viscosity solution of the first equation in (I.1) and that $m$ is a probability density solving the second equation in the sense of distributions. The model (I.1) describes the equilibrium for a population of agents with that have difficulty moving in a high-density region. Each agent faces a deterministic control problem with dynamics

This work was partially supported by KAUST baseline and startup funds and KAUST SRI, Uncertainty Quantification Center in Computational Science and Engineering.

D. Gomes, L. Nurbekyan and M. Prazeres are with the King Abdullah University of Science and Technology (KAUST), CEMSE Division and KAUST SRI, Uncertainty Quantification Center in Computational Science and Engineering, Thuwal 23955-6900, Saudi Arabia.

levon. nurbekyan@kaust.edu.sa,

mariana.prazeres@kaust.edu.sa. $\dot{x}=v$, where $v$ is a control, and seeks to minimize

$$
\begin{aligned}
& \int_{-T}^{T} m(x(s), s)^{\alpha}\left(\frac{v(s)^{2}}{2}-p v(s)\right) \\
& -V(x(s))+g(m(x(s), s)) d s+u(x(T), T) .
\end{aligned}
$$

Agents act optimally, and their collective actions drive the evolution of $m$. The corresponding time-dependent MFG is

$$
\left\{\begin{array}{l}
-u_{t}+\frac{\left(u_{x}+p\right)^{2}}{2 m^{\alpha}}+V(x)=g(m(x)) \\
m_{t}-\left(m^{1-\alpha}\left(u_{x}+p\right)\right)_{x}=0
\end{array}\right.
$$

Usually, we consider the previous system with initial-terminal conditions $m(x,-T)$ and $u(x, T)$. If $(u, m, \bar{H})$ solves (I.1) then $(u-\bar{H} t, m)$ solves (I.3). Stationary problems such as (I.1) arise in the limit, $T \rightarrow \infty$, of (I.3).

Note that the larger is $\alpha$, the smaller is the integrand in (I.2) for small values of $m$. Since $m$ is the distribution of agents, these trying to minimize (I.2) are attracted to regions where $m$ is small. Hence, $\alpha$ measures the attraction (thus, congestion) strength. Furthermore, the closer agents are to a maximum of $V$ the smaller is the integrand in (I.2). Therefore, agents prefer to stay close to maximal values of $V$.

For second-order congestion problems and a small enough $T$, the existence of a solution to (I.3) was established in [15] and [16] (resp. strong and weak solutions). For second-order problems, existence of stationary solutions was proven in [6]. In the stationary setting, existence and regularity of MFGs without congestion were considered in [10], [12], [14], [22], [23] (strong solutions) and [5], [19] (weak solutions). Many other stationary problems are examined in the literature, including obstacle problems [9], weaklycoupled systems [8], multi-populations [4], and logistic problems [13]. MFGs on networks, see [1], [3], [2], are important cases of one-dimensional MFGs.

There are few known explicit solutions for MFG (see [11] for a partial list). In the case of MFG with congestion, existence is only known for second-order problems (see [6]). Besides, the case $\alpha>2$ does not satisfy the monotonicity condition of Lasry and Lions. Hence, uniqueness may not hold. Thus, there is a keen 
interest in understanding the corresponding solutions. Here, we adapt a technique from [7]. Solutions obtained here give important insight on the regularity and existence of solutions for more general congestion MFGs.

Here, we analyze the cases where $\alpha<2$, the transition case $\alpha=2$, and $\alpha>2$, with $g$ increasing or decreasing. For $\alpha<2$ with $g$ increasing and $\alpha>2$ with $g$ decreasing, the solutions are unique and smooth. In contrast, in the remaining cases, discontinuities or nonexistence of solution can occur. Moreover, we study the existence of unhappiness traps; that is, solutions concentrated near the minimum of $V$, the less desirable regions according to (I.2). This paper is organized as follows. First, we develop the current formulation in Section III and use it to analyze (I.1). Next, in Section III we examine various examples that depict the possible behavior of the solutions. Afterward, in Section IV, we briefly consider the case of a vanishing current. We end the paper with concluding remarks in Section V

\section{Current Formulation}

To solve (I.1), we define the current $j$ as

$$
j=m^{1-\alpha}\left(u_{x}+p\right) .
$$

From the second equation in (I.1), $j$ is a constant. Here, we assume $j>0$ for definiteness. The case $j<0$ is analogous. We consider the case $j=0$ in Section IV

For $j>0$, we use (II.1) to rewrite (I.1) as

$$
\left\{\begin{array}{l}
\frac{j^{2}}{2 m^{2-\alpha}}-g(m(x))=\bar{H}-V(x) \\
\int m=1, m \geqslant 0 .
\end{array}\right.
$$

Here, we consider two model cases. First, the monotone increasing case with $g(m)=m$. Then, the monotone decreasing case with $g(m)=-m$. We made these choices for definiteness but our methods are valid for a general monotone $g$, increasing or decreasing.

\section{A. Case I. $\alpha<2$ and $g(m)=m$}

The function on (II.2), $t \mapsto \frac{j^{2}}{2 t^{2-\alpha}}-t$, is strictly decreasing. To find $m$, we solve (II.2) and select $\bar{H}$ such that $\int_{\mathbb{T}} m=1$.

\section{B. Case II. $\alpha<2$ and $g(m)=-m$}

Here, the function on (II.2), $t \mapsto \frac{j^{2}}{2 t^{2-\alpha}}+t:=G_{\alpha}(t)$, is not invertible, and it has a minimum for $t_{\min }=$ $\left(j^{2}-\frac{\alpha j^{2}}{2}\right)^{\frac{1}{3-\alpha}}$. Hence, for

$$
\bar{H} \geqslant G_{\alpha}\left(t_{\min }\right)+\max _{\mathbb{T}} V=\bar{H}_{c r},
$$

the first equation of (II.2) has two solutions for each $x \in \mathbb{T}, m_{\bar{H}}^{+}(x)$ and $m_{\bar{H}}^{-}(x)$, where $m_{\bar{H}}^{-}(x) \leqslant t_{\text {min }} \leqslant$
$m_{\frac{+}{H}}^{+}(x)$.

Next, we define the quantities

$$
\left\{\begin{array}{l}
\phi^{+}=\int_{\mathbb{T}} m_{\bar{H}_{c r}}^{+}(x) \\
\phi^{-}=\int_{\mathbb{T}} m_{\bar{H}_{c r}}^{-}(x) .
\end{array}\right.
$$

Because $\phi^{-} \leqslant \phi^{+}$, we have three alternatives

1. $\phi^{+} \leqslant 1$;

2. $\phi^{-} \geqslant 1$

3. $\phi^{-}<1<\phi^{+}$.

In the first alternative, because the function $G_{\alpha}$ is strictly increasing in $\left(t_{\min },+\infty\right)$, there exists $\bar{H}$ such that $\int_{\mathbb{T}} m_{\bar{H}}^{+}(x)=1$. Hence, $m(x)=m_{\bar{H}}^{+}(x)$. In the second alternative, because the function $G_{\alpha}$ is strictly decreasing in $\left(0, t_{\text {min }}\right)$, there exists $\bar{H}$ such that $\int_{\mathbb{T}} m_{\bar{H}}^{+}(x)=1$. Thus, $m(x)=m_{\bar{H}}^{-}(x)$. Finally, in the last alternative, when $\phi^{-}<1<\phi^{+}$, the solution $m$ takes the values $m_{\bar{H}}^{+}$and $m_{\bar{H}}^{-}$for some $\bar{H} \geqslant \bar{H}_{c r}$. We consider viscosity solutions $u$ that are semiconcave. Then, $\bar{H}=\bar{H}_{c r}$ (see [7]) and $m$ can switch from $m_{\bar{H}}^{+}$ to $m_{\bar{H}}^{-}$at the maxima of $V$. This reasoning leads to:

Proposition 1: Let $g(m)=m$. Suppose $V$ is not constant and has a single maximum at $x=0$. Then, for every $j>0$, there is a unique triplet $(u, m, \bar{H})$ that solves (II.2). Moreover,

$$
u(x)=\int_{0}^{x} \frac{j d y}{m(x)}-p x, p=\int_{\mathbb{T}} \frac{j d y}{m(x)}
$$

and if $\phi^{+} \leqslant 1, m(x)=m_{\frac{H}{H}}^{+}(x)$ and if $\phi^{-} \geqslant 1$, $m(x)=m_{\bar{H}}^{-}(x)$. In both cases, $\bar{H}$ is such that $\int m=$ 1. If $\phi^{-}<1<\phi^{+}, \bar{H}=\bar{H}_{c r}$ and $m(x)=$ $m_{\bar{H}_{c r}}^{-}(x) \chi_{[0, d]}+m_{\bar{H}_{c r}}^{+}(x) \chi_{[d, 1]}$, where $d$ is such that $\int_{0}^{d} m_{\bar{H}}^{-}(x)+\int_{d}^{1} m_{\bar{H}}^{+}(x)=1$.

By the preceding proposition, we recover the solution either by numerical or explicit integration in (II.5).

C. Case III. $\alpha>2$ and $g(m)=-m$

Because $\alpha>2$, we write (II.2) as

$$
\left\{\begin{array}{l}
\frac{j^{2}}{2} m^{\alpha-2}+m=\bar{H}-V(x) \\
\int m=1, m \geqslant 0 .
\end{array}\right.
$$

The function on the left-hand side of (II.6),

$$
t \rightarrow \frac{j^{2}}{2} t^{\alpha-2}+t
$$

is strictly increasing for $t>0$. We find $m$ solving (II.6) and selecting $\bar{H}$ such that $\int_{\mathbb{T}} m=1$. However, the condition $m>0$ may fail, and, thus, a non-negative solution may not exist. 
D. Case IV. $\alpha>2$ and $g(m)=m$

The function on the left-hand side of (II.6),

$$
t \rightarrow \frac{j^{2}}{2} t^{\alpha-2}-t:=F_{\alpha}(t),
$$

is not monotone. Because $F_{\alpha}^{\prime \prime}(t)=(\alpha-2)(\alpha-$ 3) $\frac{j^{2}}{2} t^{\alpha-4}$, we see that, for $2<\alpha<3, F$ has a maximum in $t_{\max }$ and for $\alpha>3, F$ has a minimum in $t_{\text {min }}$. A simple computation yields

$$
t_{\text {max }}, t_{\text {min }}=\left(\frac{\alpha j^{2}}{2}-j^{2}\right)^{\frac{1}{3-\alpha}} .
$$

For $g(m)=m$, III.6 is degree $\max \{\alpha-2,1\}$ polynomial. Thus, the transition at $\alpha=3$.

1) Case IV (a). $2<\alpha<3$ : In this case, $F$ has a maximum. Hence, if

$$
\bar{H} \leqslant F_{\alpha}\left(t_{\max }\right)+\min _{\mathbb{T}} V=\bar{H}_{c r}^{\max },
$$

the first equation of (II.6) has two solutions for each $x \in \mathbb{T}, m_{\bar{H}}^{+}$and $m_{\bar{H}}^{-}$, where $m_{\bar{H}}^{-} \leqslant t_{\max } \leqslant m_{\bar{H}}^{+}$.

2) Case $I V(b) . \alpha>3$ : In this case, $F$ has a minimum. Thus, if

$$
\bar{H} \geqslant F_{\alpha}\left(t_{m i n}\right)+\max _{\mathbb{T}} V=\bar{H}_{c r}^{m i n},
$$

the first equation in III.6 has two solutions, $m_{\bar{H}}^{+}$and $m_{\bar{H}}^{-}$with $m_{\bar{H}}^{-} \leqslant t_{\min } \leqslant m_{\bar{H}}^{+}$.

The reasoning in Section II.B does not depend on whether $t_{\min }$ is a maximum or a minimum. Thus, arguing as before, we get the following proposition.

Proposition 2: Let $g(m)=m$. Suppose $V$ is not constant and has a single maximum at $x=0$. Then, for every current level $j>0$ there is a unique triplet $(u, m, \bar{H})$ that solves (II.2) with $g(m)=m$. If $\phi^{+} \leqslant 1$, $m(x)=m_{\frac{H}{H}}^{+}(x)$ and if $\phi^{-} \geqslant 1, m(x)=m_{\bar{H}}^{-}(x)$. In both cases, $\bar{H}$ is such that $\int m=1$. If $\phi^{-}<1<\phi^{+}$ and $2<\alpha<3$, let $\bar{H}=\bar{H}_{c r}^{\text {max }}$, otherwise, for $\alpha>3$, $\bar{H}=\bar{H}_{c r}^{\min }$. Finally, let $d$ be such that

$$
\int_{0}^{d} m_{\bar{H}}^{-}(x)+\int_{d}^{1} m_{\bar{H}}^{+}(x)=1 .
$$

Then, solving the above equation for $d$, we get the representation formula

$$
m(x)=m_{\bar{H}}^{-}(x) \chi_{[0, d]}+m_{\bar{H}}^{+}(x) \chi_{[d, 1]},
$$

where $\chi_{A}$ represents the indicator function of $A$ and $\bar{H}$ takes the corresponding value. Moreover,

$$
u(x)=\int_{0}^{x} \frac{j d y}{m(x)}-p x, p=\int_{\mathbb{T}^{d}} \frac{j d y}{m(x)} .
$$

Note that the solutions given in the preceding proposition may fail to be non-negative, as we illustrate in Section $\amalg$.
3) Case IV (c). $\alpha=3$ : For $\alpha=3$, (II.6) becomes

$$
\left\{\begin{array}{l}
\frac{j^{2}}{2} m-m=\bar{H}-V(x) \\
\int m=1, m \geqslant 0 .
\end{array}\right.
$$

Thus,

$$
m(x)=\frac{\bar{H}-V(x)}{\frac{j^{2}}{2}-1} \text { for } x \in \mathbb{T},
$$

and

$$
\bar{H}=\frac{j^{2}}{2}-1+\int_{\mathbb{T}} V
$$

solve the first equation in (II.14). However, we need $m \geqslant 0$ to hold. Thus, we have existence of nonnegative solutions in two cases:

1. $j \leqslant \sqrt{2}$ and $\frac{j^{2}}{2}-1+\int_{\mathbb{T}} V \leqslant \min V$,

2. If $j \geqslant \sqrt{2}$ and $\frac{j^{2}}{2}-1+\int_{\mathbb{T}} V \geqslant \max V$.

E. Case V. $\alpha=2$

The equation (II.6) becomes

$$
\left\{\begin{array}{l}
\frac{j^{2}}{2}+V(x)=g(m(x))+\bar{H} \\
\int m=1, m \geqslant 0,
\end{array}\right.
$$

and we have the following proposition.

Proposition 3: Suppose $g$ has a monotone inverse $g^{-1}$ and that

$$
g^{-1}\left(\frac{j^{2}}{2}+V(x)-\bar{H}\right) \geqslant 0 .
$$

Then, for every $j>0$, there is a unique solution $(u, m, \bar{H})$ that solves (II.17). Moreover,

$$
u(x)=\int_{0}^{x} \frac{j d y}{m(x)}-p x, p=\int_{\mathbb{T}^{d}} \frac{j d y}{m(x)},
$$

and

$$
m(x)=g^{-1}\left(\frac{j^{2}}{2}+V(x)-\bar{H}\right) \text { for } x \in \mathbb{T},
$$

where $\bar{H}$ is such that $\int m=1$.

\section{Results AND ANALYSis}

Here, we illustrate the previous examples.

A. Case I. $\alpha<2$ and $g(m)=m$

In this first case, (I.1) satisfies the Lasry-Lions monotonicity condition. Because of the analysis in the preceding Section, the solution is unique and $C^{\infty}$ if $m>0$. We depict in Figure 1 the solution $m$, and see that low-density regimes correspond to the minimum of $V$, as expected in face of (I.2). 


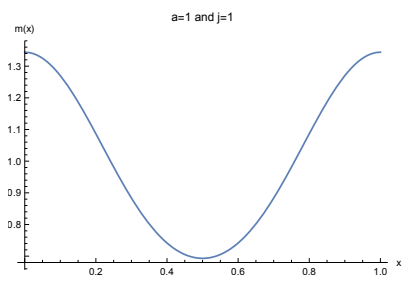

Fig. 1. Solution $m$ for $\alpha=1, j=1, V(x)=\frac{1}{2} \sin (2 \pi(x+1 / 4))$, and $g(m)=m$.

\section{B. Case II. $\alpha<2$ and $g(m)=-m$}

Here, as shown in Section II, there are three cases. Figure 2 illustrates the case, $\phi^{+} \leqslant 1$. The solution is $C^{\infty}$, and high-density regimes correspond to an unhappiness trap near the minimum of $V$. This surprising behavior is caused by decreasing $g$ - agents want to stick together. Figure 4 illustrates the case $\phi^{-} \geqslant 1$. Here, the solution is $C^{\infty}$, and as $j \rightarrow \infty$, $m \rightarrow 1$. Finally, in Figure 3 , $\phi^{-}<1<\phi^{+}$. Here, $m$ is discontinuous and switches between high and lowdensity regimes near to the minimum of $V$.

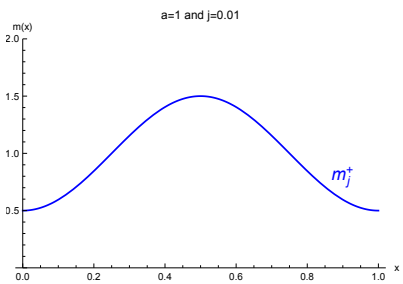

Fig. 2. Solution $m$ for $\alpha=1, j=0.01, V(x)=\frac{1}{2} \sin (2 \pi(x+$ $1 / 4))$, and $g(m)=-m$.

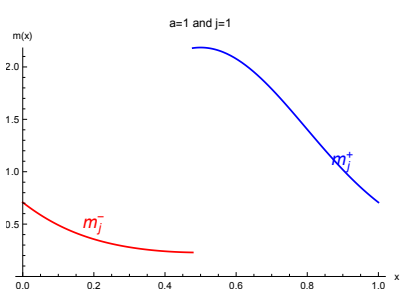

Fig. 3. Solution $m$ for $\alpha=1, j=1, V(x)=\frac{1}{2} \sin (2 \pi(x+1 / 4))$, and $g(m)=-m$.

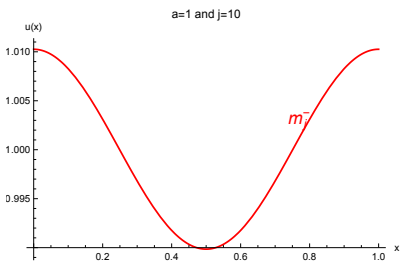

Fig. 4. Solution $m$ for $\alpha=1, j=10, V(x)=\frac{1}{2} \sin (2 \pi(x+$ $1 / 4)$ ), and $g(m)=-m$.

C. Case III. $\alpha>2$ and $g(m)=-m$

From Section III we have a unique $C^{\infty}$ solution $m$. Figure 5 depicts $m$. In contrast with Case I, high- density areas correspond to the minimum of $V$. Again, we see unhappiness traps. However, in Figure 6 , for $V$ with high-oscillations and small current there is not a non-negative solution $m$.

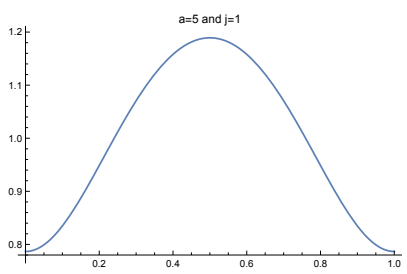

Fig. 5. Solution $m$ for $\alpha=5, j=1, V(x)=\frac{1}{2} \sin (2 \pi(x+1 / 4))$, and $g(m)=-m$.

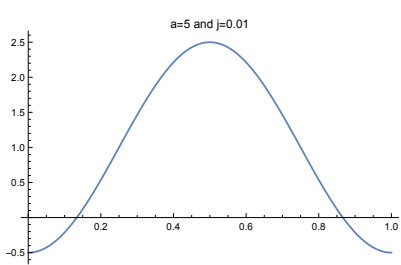

Fig. 6. Non positive solution $m$ for $\alpha=5, j=0.01, V(x)=$ $\frac{3}{2} \sin (2 \pi(x+1 / 4))$, and $g(m)=-m$.

D. Case IV. $\alpha>2$ and $g(m)=m$

We divide the analysis of this problem into two cases, $2<\alpha<3$ and $\alpha>3$, according to which term dominates in II.6 We omit the analysis of the transition case $\alpha=3$.

1) Case IV (a). $2<\alpha<3$ : Here, we have three cases. In Figure 7, $\phi^{+} \leqslant 1$, and we observe low-density regimes in the minimum of $V$. Next, in Figure $9, \phi^{-} \geqslant$ 1 , we observe that $m$ becomes closer to 1 as the current is large. Also, in contrast to the case II, unhappiness traps arise in the high-current regime. Finally, in Figure 8. we have the remaining case, $\phi^{-}<1<\phi^{+}$. The solution $m$ exhibits a discontinuity with a transition between high and low-density near to the minimum of $V$.

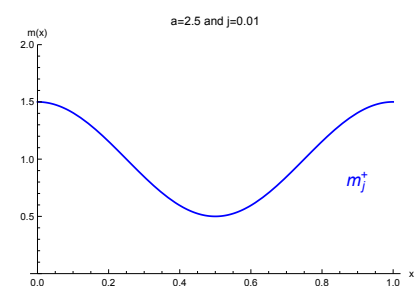

Fig. 7. Solution $m$ for $\alpha=2.5, j=0.01, V(x)=\frac{1}{2} \sin (2 \pi(x+$ $1 / 4))$, and $g(m)=-m$. 


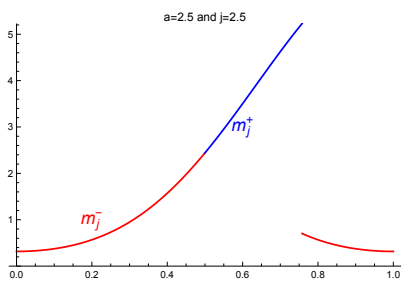

Fig. 8. Solution $m$ for $\alpha=2.5, j=2.5, V(x)=\frac{1}{2} \sin (2 \pi(x+$ $1 / 4)$ ), and $g(m)=-m$.

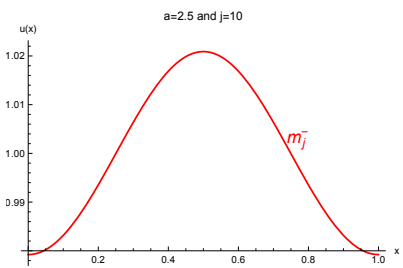

Fig. 9. Solution $m$ for $\alpha=2.5, j=10, V(x)=\frac{1}{2} \sin (2 \pi(x+$ $1 / 4))$, and $g(m)=-m$.

2) Case IV (b). $\alpha>3$ : There are three cases. First, in Figure $10, \phi^{+} \leqslant 1$ and we observe low-density regimes at the minimum of $V$. Next, in Figure 13 $\phi^{-} \geqslant 1$ and $m$ approaches 1 as the current increases. Finally, in Figures 12 and 11, $\phi^{-}<1<\phi^{+}$. In both situations, $m$ is discontinuous, so we have both high and low-density regimes next to the minimum of $V$. But, in Figure 11, we observe a non-positive solution, so we don't have existence.

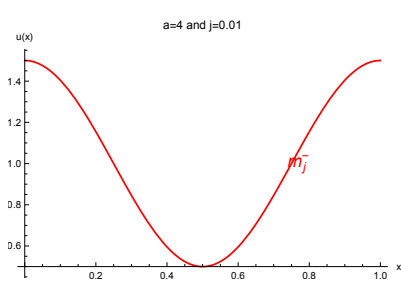

Fig. 10. Solution $m$ for $\alpha=4, j=0.01, V(x)=\frac{1}{2} \sin (2 \pi(x+$ $1 / 4))$, and $g(m)=m$.

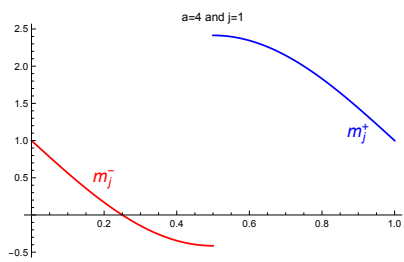

Fig. 11. Non-positive solution $m$ for $\alpha=4, j=1, V(x)=$ $\frac{1}{2} \sin (2 \pi(x+1 / 4))$, and $g(m)=m$.

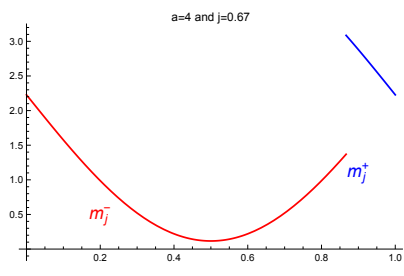

Fig. 12. Solution $m$ for $\alpha=4, j=0.67, V(x)=\frac{1}{2} \sin (2 \pi(x+$ $1 / 4)$ ), and $g(m)=m$.

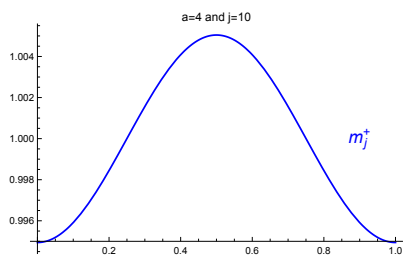

Fig. 13. Solution $m$ for $\alpha=4, j=10, V(x)=\frac{1}{2} \sin (2 \pi(x+$ $1 / 4))$, and $g(m)=m$.

E. Case V. $\alpha=2$ for both $g$ increasing and decreasing.

In this last case, $m$ is unique and $C^{\infty}$ for $g(m)=$ $\pm m$. Figure 14 depicts an unhappiness trap, highdensity near the minimum of $V$. Figure 15 shows a low-density near the minimum.

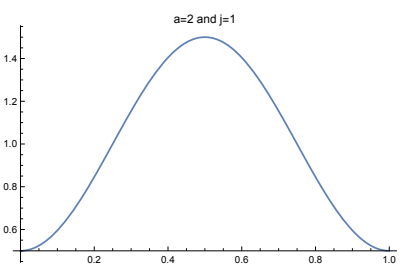

Fig. 14. Solution $m$ for $\alpha=2, \forall j, V(x)=\frac{1}{2} \sin (2 \pi(x+1 / 4))$, and $g(m)=-m$.

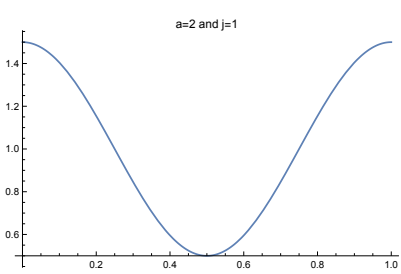

Fig. 15. Solution $m$ for $\alpha=2, \forall j, V(x)=\frac{1}{2} \sin (2 \pi(x+1 / 4))$, and $g(m)=m$.

\section{ZERO CURRENT}

Here, we set $j=0$. Then, I.1P becomes

$$
\left\{\begin{array}{l}
\frac{\left(u_{x}+p\right)^{2}}{2 m^{\alpha}}-g(m(x))=\bar{H}-V(x) \\
-m^{1-\alpha}\left(u_{x}+p\right)=0 .
\end{array}\right.
$$

If $m=0$, we get $u_{x}+p=0$ because of the congestion term in the first equation of (IV.1). If $m>0$, the second equation in (IV.1) gives $u_{x}+p=0$. Consequently, $u_{x}+p=0$ everywhere. Thus, we get the following 
result.

Proposition 4: Suppose $g$ is invertible. Suppose there exists $\bar{H}$ such that

$$
m=g^{-1}(V(x)-\bar{H}) \geqslant 0
$$

satisfies $\int m d x=1$. Then, $p=0$ is the unique value for which there exists a zero current solution of (IV.1). Hence, $(0, m, \bar{H})$ is the corresponding unique solution with the normalization condition $\int u=0$.

\section{Conclunsions}

We explored the one-dimensional structure of MFGs and the current formulation to obtain solutions of firstorder MFGs with congestion. In problems without congestion, the theory is relatively well understood. In contrast, in congestion problems, the existence of smooth solutions was only known for certain secondorder problems [6], and the existence of weak solutions was only known under monotonicity conditions [5]. Here, we have given examples of first-order MFGs for which smooth solutions exist, where only weak solutions exist, and where no solutions exist. The asymptotic behavior as $j \rightarrow \infty$ was performed in [7], where it was shown that $m \rightarrow 1$ for problems without congestion. There, the uniqueness question was also addressed. Similar results hold for the congestion case. Even for monotone problems, uniqueness may fail and depends on properties of the potential $V$ [7]. The solutions computed here may prove useful to guide future research directions, in applications to MFGs on graphs, and for the validation of numerical methods.

\section{REFERENCES}

[1] D. Bauso, X. Zhang, and A. Papachristodoulou. Density flow over networks: A mean-field game theoretic approach. volume 2015-February, pages 3469-3474, 2014.

[2] S. Cacace, F. Camilli, and C Marchi. A numerical method for mean field games on networks. preprint, 2015.

[3] F. Camilli, E. Carlini, and C Marchi. A model problem for mean field games on networks. Discrete Contin. Dyn. Syst., 35(9):4173-4192, 2015.

[4] M Cirant. Multi-population mean field games systems with Neumann boundary conditions. To appear in J. Math. Pures Appl., 2014.

[5] R. Ferreira and D. Gomes. Existence of weak solutions for stationary mean-field games through variational inequalities. Preprint.

[6] D. Gomes and H. Mitake. Existence for stationary meanfield games with congestion and quadratic Hamiltonians. NoDEA Nonlinear Differential Equations Appl., 22(6):18971910, 2015.

[7] D. Gomes, L. Nurbekyan, and M. Prazeres. Explicit solutions of one-dimensional first-order stationary mean-field games with a generic nonlinearity. Preprint, 2016.

[8] D. Gomes and S. Patrizi. Weakly coupled mean-field game systems. Preprint.

[9] D. Gomes and S. Patrizi. Obstacle mean-field game problem. Interfaces Free Bound., 17(1):55-68, 2015.

[10] D. Gomes, S. Patrizi, and V. Voskanyan. On the existence of classical solutions for stationary extended mean field games. Nonlinear Anal., 99:49-79, 2014.
[11] D. Gomes, E. Pimentel, and V. Voskanyan. Regularity theory for mean-field game systems. 2016.

[12] D. Gomes, G. E. Pires, and H. Sánchez-Morgado. A-priori estimates for stationary mean-field games. Netw. Heterog. Media, 7(2):303-314, 2012.

[13] D. Gomes and R. Ribeiro. Mean field games with logistic population dynamics. 52nd IEEE Conference on Decision and Control (Florence, December 2013), 2013.

[14] D. Gomes and H. Sánchez Morgado. A stochastic EvansAronsson problem. Trans. Amer. Math. Soc., 366(2):903-929, 2014.

[15] D. Gomes and V. Voskanyan. Short-time existence of solutions for mean-field games with stion. To appear J. London Math. Soc.

[16] J. Graber. Weak solutions for mean field games with congestion. Preprint, 2015.

[17] M. Huang, P. E. Caines, and R. P. Malhamé. Largepopulation cost-coupled LQG problems with nonuniform agents: individual-mass behavior and decentralized $\epsilon$-Nash equilibria. IEEE Trans. Automat. Control, 52(9):1560-1571, 2007.

[18] M. Huang, R. P. Malhamé, and P. E. Caines. Large population stochastic dynamic games: closed-loop McKean-Vlasov systems and the Nash certainty equivalence principle. Commun. Inf. Syst., 6(3):221-251, 2006

[19] J.-M. Lasry and P.-L. Lions. Jeux à champ moyen. I. Le cas stationnaire. C. R. Math. Acad. Sci. Paris, 343(9):619-625, 2006.

[20] J.-M. Lasry and P.-L. Lions. Jeux à champ moyen. II. Horizon fini et contrôle optimal. C. R. Math. Acad. Sci. Paris, 343(10):679-684, 2006.

[21] J.-M. Lasry and P.-L. Lions. Mean field games. Jpn. J. Math., 2(1):229-260, 2007.

[22] E. Pimentel and V. Voskanyan. Regularity for second-order stationaty mean-field games. To appear in Indiana University Mathematics Journal.

[23] V. K. Voskanyan. Some estimates for stationary extended mean field games. Dokl. Nats. Akad. Nauk Armen., 113(1):30-36, 2013. 